\title{
Closed orbit instability
}

Viatcheslav Danilov, Stuart Henderson, and Jeff Holmes
Oak Ridge National Laboratory, Oak Ridge, Tennessee 37830

A. Burov

Fermi National Laboratory, Batavia, Illinois 60510

(Received 17 October 2001; published 11 December 2001)

\begin{abstract}
Because of a long-range resistive wake, the closed orbit may experience an unstable drift. Unlike the conventional betatron instabilities, this closed orbit instability is not sensitive to the spread of the betatron frequencies. For bunched beams, feedback appears to be the only way to stabilize the closed orbit above threshold. This new instability can be significant for both existing and designed high-intensity rings.
\end{abstract}

DOI: 10.1103/PhysRevSTAB.4.120101

PACS numbers: 29.27.Bd, 29.27.Fh

\section{INTRODUCTION}

In the course of collective stability analysis, normally only eigenfrequencies near betatron sidebands of the revolution frequency are considered. Imagine, however, that a wake function, such as a resistive wall wake, has a very long tail, much longer than a revolution period. In this case, the wake fields are accumulated over a long time if the closed orbit is distorted. This accumulated field generates further effective dipole field error and leads to an unstable drift of the closed orbit. An estimation of this effect shows that it could be very important for high-intensity proton rings, and for electron rings with a large full current, such as B factories.

\section{BASIC PHYSICS}

To begin, consider the simple model of a single macroparticle inside a homogeneous chamber. Taking into account the accumulation of the wake field, the transverse equation of motion can be written as (see, e.g., [1])

$$
\ddot{y}+\omega_{b}^{2} y=-\frac{N r_{0} c^{2}}{\gamma \Pi} \sum_{k=1}^{\infty} y(t-k T) W(-k \Pi),
$$

where $N$ is the number of particles, $r_{0}$ is the particle's classical radius, $W(z)$ is the wake function, $\Pi=c T$ is the storage ring circumference, $\omega_{b}$ is the betatron frequency, and the remaining constants correspond to the standard physical notation. With $y(t) \propto \exp (-i \omega t)$, Eq. (1) leads to

$$
-\omega^{2}+\omega_{b}^{2}=-\frac{N r_{0} c^{2}}{\gamma \Pi} \sum_{k=1}^{\infty} W(-k \Pi) \exp (i k \omega T) .
$$

Conventional betatron eigenfrequencies are found as solutions at $\omega \approx \pm \omega_{b}$. Far more important for the present discussion is the fact that Eq. (2) has additional solutions that can be significant for long-range wakes, by which we mean $|W(s-T)-W(s)| \ll|W(s)|$. Let us assume that Eq. (2) has a solution in the vicinity of a multiple of the revolution frequency $\omega=n \omega_{0}+\Omega$, with $|\Omega| \ll \omega_{0}$. Then, the rapid variation $n \omega_{0}$ drops out of the sum on the right-hand side of Eq. (2), and the remaining slow variation can be approximated by an integral $\sum_{k=1}^{\infty} \cdots=$ $\int_{0}^{\infty} d k \cdots=\frac{1}{T} \int_{0}^{\infty} \frac{d s}{c} \cdots$, leading to the following equation for the complex frequency shift $\Omega$ :

$$
-n^{2} \omega_{0}^{2}+\omega_{b}^{2}=i \frac{N r_{0} c}{\gamma T^{2}} Z_{\perp}(\Omega),
$$

where the slow variation $\Omega$ is dropped from the left-hand side.

Because the wake function $W(s)=0$ for $s>0$, all the singularities of the transverse impedance $Z_{\perp}(\Omega)=$ $i \int_{0}^{\infty} \frac{d s}{c} \exp (i \Omega s / c) W(-s)$ lie in the lower half-plane of its argument, at $\operatorname{Im} \Omega<0$. Let us suppose for simplicity that the only singularity is a simple pole at $\Omega=-i \lambda$, so that

$$
\begin{aligned}
Z_{\perp}(\Omega) & =-W_{0} /(\Omega+i \lambda) \\
& \Leftrightarrow W(-s)=W_{0} \exp (-\lambda s / c) .
\end{aligned}
$$

In this case, the dispersion equation (3) yields

$$
\Omega=-i \lambda-i \frac{N r_{0} c W_{0}}{4 \pi^{2} \gamma\left(\nu_{b}^{2}-n^{2}\right)},
$$

where $\nu_{b}$ is the betatron tune. There are several interesting features of this solution. First, it describes a term in an infinite sequence of eigenfrequencies, indexed by the arbitrary integer $n$. In fact, all these modes describe the multiturn dynamics of the wake fields, and the number $n$ is associated with the $n$th spatial harmonic of the wake field expansion over the ring azimuth $\theta=2 \pi s / \Pi$. When the number of particles is small enough, all these modes decay as the field decrement $\lambda$, some of them being slightly faster, and some slightly slower. The value $-i \lambda$ can be considered as a single-particle "frequency" of the drift modes, while the intensity-dependent part in the right-hand side of Eq. (5) gives their coherent "tune shifts." When the beam intensity increases, the decrement of the most long-lived mode approaches zero, and at a certain current this mode becomes unstable. This threshold intensity is calculated from Eq. (5). To determine the threshold, assume, as is usual, 
that the wake amplitude $W_{0}$ is negative, $W_{0}=-\left|W_{0}\right|$. In this case, the number of the least stable mode is equal to the integer part of the betatron tune $n=\left[\nu_{b}\right]$, and the threshold intensity $N_{\text {th }}$ is given by

$$
\frac{N_{\mathrm{th}} r_{0} c\left|W_{0}\right|}{8 \pi^{2} \gamma \nu_{b}\left\{\nu_{b}\right\} \lambda}=1,
$$

where $\left\{\nu_{b}\right\}=\nu_{b}-\left[\nu_{b}\right]$ is the fractional betatron tune. Note that the threshold is determined by the amplitude of the integral wake $c W_{0} / \lambda=\int_{0}^{\infty} d s W(-s)$, which reflects the specific nature of this instability that it is caused by the long-term accumulation of the wake fields, leading to a drift of the closed orbit. Hence, it follows that this closed orbit instability is determined by the total number of particles in the ring $N$, and is insensitive to their bunching, so long as $|\Omega| \ll \omega_{0}$. In other words, the same dispersion equation (5) and threshold condition (6) describe singlebunch, multibunch, and coasting beams.

One further important point is the independence of these drift modes on the spread of the betatron frequencies. For bunched beams, neither the spread of the revolution frequencies nor synchrotron motion matter. For a coasting beam, however, the spread of the revolution frequencies $\Delta \omega_{0}$ damps the closed orbit instability if $n \Delta \omega_{0}>|\Omega|$.

It is interesting to compare the closed orbit and the betatron mode increments. For a coasting beam, the coherent betatron tune shift $\Omega_{b}$ of the $n$th azimuthal mode driven by the same exponential wake (4) is

$$
\Omega_{b}=i \frac{N r_{0} c W_{0}}{8 \pi^{2} \gamma \nu_{b}\left(\nu_{b}-n\right)}
$$

where the wake decrement was assumed to be small enough, $\lambda \ll \omega_{0}\left\{\nu_{b}\right\}$. The betatron tune shift (7) is almost exactly opposite to the coherent tune shift of the closed orbit mode of the same number, given by Eq. (5). This means that the stability conditions for these two types of modes are opposite: where the betatron mode is most stable, the drift mode is most unstable and vice versa. Note that this equality of the coherent parts of the betatron and drift modes is specific to the exponential wake function, introduced above as a simple model. In fact, however, this exponential function is more than just a simple model: It describes a resistive wall wake in the long-range limit, as we discuss below.

\section{DRIFT EQUATION IN GENERAL CASE}

In this section, we derive an equation for drift modes with arbitrary impedance and lattice functions. As was pointed out above, the behavior of the drift modes does not depend on the beam bunching. Here, the calculations are carried out for a coasting beam. Then, for the specific case of a homogeneous wake function and smooth focusing, the result is shown to be identical to the macroparticle beam of the previous section, Eq. (3).

To begin, consider the case of a long-range wake function generated by a localized element at a specific azimuth of the storage ring. This is opposite to the previous section, where the wake function was assumed to be homogeneous around the orbit. At the location $s^{\prime}$, where the wake field is located, each particle is kicked by an angle

$$
\vartheta\left(s^{\prime}, t\right)=-\frac{N r_{0}}{\gamma \Pi} \int_{0}^{\infty} d z W(-z) y\left(s^{\prime}, t-z / c\right) .
$$

This angle causes a distortion of the closed orbit (see, e.g., [2]),

$$
y(s, t)=\vartheta\left(s^{\prime}, t\right) \frac{\sqrt{\beta(s) \beta\left(s^{\prime}\right)} \cos \left[\pi \nu-\psi\left(s, s^{\prime}\right)\right]}{2 \sin (\pi \nu)},
$$

where $\beta(s)$ is the lattice beta function and $\psi\left(s, s^{\prime}\right)$ is the betatron phase advance between the points $s^{\prime}$ and $s$ determined so that $0<\psi\left(s, s^{\prime}\right)<2 \pi \nu$.

Assuming $y(s, t)=y(s) \exp (-i \Omega t)$, the closed orbit equation leads to

$$
\begin{aligned}
y(s)= & \frac{i N r_{0} Z_{\perp}(\Omega)}{\gamma T} \frac{\sqrt{\beta(s) \beta\left(s^{\prime}\right)} \cos \left[\pi \nu-\psi\left(s, s^{\prime}\right)\right]}{2 \sin (\pi \nu)} \\
& \times y\left(s^{\prime}\right),
\end{aligned}
$$

which in turn gives rise to the dispersion equation

$$
1=\frac{i N r_{0} Z_{\perp}(\Omega) \beta\left(s^{\prime}\right)}{2 \gamma T} \cot (\pi \nu) .
$$

Note that, for the drift modes, the dispersion relation depends on both the impedance and the local $\beta$ function, as it does for the conventional betatron modes. In distinction from the homogeneously distributed impedance of Eq. (3), this localized impedance gives only a finite number of solutions, namely, one, in the case of the exponential wake treated above. The reason for this, for the localized wake source, is that the fields have a finite number of degrees of freedom.

It is now simple to write an equation for an arbitrary impedance distribution around the ring. Because of linearity, all the closed orbit perturbations can be directly summed. Thus, if $\tilde{Z}_{\perp}\left(s^{\prime}, \Omega\right)$ is defined to be a linear density of impedance at the azimuth $s^{\prime}$, so that the impedance of the length $d s^{\prime}$ is $d Z_{\perp}\left(s^{\prime}, \Omega\right)=\tilde{Z}_{\perp}\left(s^{\prime}, \Omega\right) d s^{\prime}$, the equation for the periodic eigenfunctions $y(s)$ and eigenvalues $\Omega$ is

$$
y(s)=\frac{i N r_{0}}{2 \gamma T \sin (\pi \nu)} \oint d s^{\prime} \tilde{Z}_{\perp}\left(s^{\prime}, \Omega\right) \sqrt{\beta(s) \beta\left(s^{\prime}\right)} \cos \left[\pi \nu-\psi\left(s, s^{\prime}\right)\right] y\left(s^{\prime}\right) .
$$


It is straightforward to show that Eq. (12) reduces to Eq. (3) in the homogeneous case of the previous section with $\beta(s)=\Pi /(2 \pi \nu)$ and $\tilde{Z}_{\perp}\left(s^{\prime}, \Omega\right)=Z_{\perp}(\Omega) / \Pi$.

\section{IMPEDANCE OF RESISTIVE WALL}

Conventionally, the resistive wall impedance is calculated assuming that the skin depth $\delta=c / \sqrt{2 \pi \sigma \Omega}$ is much smaller than the wall thickness $d$. This assumption can be incorrect for slow drift modes, and especially for a thin metallic coating, where the skin depth can be larger than the metal thickness. For instance, the skin depth in stainless steel $\left(\sigma=1.3 \times 10^{16} \mathrm{~s}^{-1}\right)$ at frequency $\Omega /(2 \pi)=1 \mathrm{KHz}$ is $\delta=1.3 \mathrm{~cm}$, while the metal thickness normally does not exceed a few millimeters.

Recently, Lebedev proposed an effective method to calculate the resistive transverse impedance of multilayer chambers [3]. Below, the results obtained by his method are presented for two model cases. For infinite vacuum outside the metal chamber, the impedance per unit length is found to be

$$
\tilde{Z}_{\perp}(\Omega)=-i \frac{Z_{0}}{\pi b^{2}} \frac{1}{2+\kappa b \tanh (\kappa d)},
$$

where $Z_{0}=4 \pi / c=377 \Omega, b \gg d$ is the chamber radius, and $\kappa=(1-i) / \delta$. In the case when an ideal magnetic material is placed outside, the impedance does not look much different (see [4]),

$$
\tilde{Z}_{\perp}(\Omega)=-i \frac{Z_{0}}{\pi b^{2}} \frac{1}{1+\kappa b \tanh (\kappa d)} .
$$

Both of these cases can be approximated as

$$
\tilde{Z}_{\perp}(\Omega)=-i \frac{Z_{0}}{\pi b^{2}} \begin{cases}1 /(\kappa b) \propto 1 / \sqrt{-i \Omega}, & \text { for }|\delta|<d, \\ g /(1+\Omega / i \lambda), & \text { otherwise, }\end{cases}
$$

where the material factor $g=1$ and the wake decrement $\lambda=c^{2} / 4 \pi \sigma b d$ for an ideal magnetic material outside, and $g=1 / 2$ and $\lambda=c^{2} / 2 \pi \sigma b d$ for the vacuum outside. If the chamber can be considered thin $(\delta>d)$, the impedance is single pole, which corresponds to the exponential wake function considered as a model in Sec. II, with

$$
\tilde{W}_{0}=-\frac{Z_{0} g \lambda}{\pi b^{2}}
$$

and the outside factor $g \cong 0.5-1$, and the tilde means that the value is taken per unit length. Note that in this case the amplitude of the integral wake $W_{0} / \lambda$ does not depend on the chamber conductivity or thickness. Taking into account that at the threshold of instability $|\Omega| \rightarrow 0$, by definition, it follows that the impedance can be taken out of the integral equation (12) for threshold calculations. In the smooth approximation, this gives the following "universal" formula for the threshold intensity [see Eq. (6)]:

$$
N_{\mathrm{th}}=\frac{2 \pi^{2} b^{2} \gamma \nu_{b}\left\{\nu_{b}\right\}}{r_{0} \Pi g} .
$$

A paradoxical feature of this result is its independence of the specific wall properties. The reason is that, although for higher conductivity the wake function amplitude decreases, the wake field decay becomes slower, so that the wake integral does not change. However, the rate of growth of the drift instability also decreases when the conductivity increases.

\section{PRACTICAL EXAMPLES}

In this section, the closed orbit instability is examined for several high-intensity rings.

For the LER of the PEP-II B Factory with $\Pi=2.2 \mathrm{~km}$, $\nu=36.6, \gamma=6 \times 10^{3}, b=2.3 \mathrm{~cm}$ (see, e.g., Ref. [4]), the smooth approximation of Eq. (17) gives $N_{\text {th }}=1.5 \times$ $10^{14}$, compared with the design value $N=1 \times 10^{14}$. Taking into account inaccuracy of the smooth approximation, it can be concluded that the closed orbit instability might be dangerous for this ring.

For the SNS ring, with $\Pi=248 \mathrm{~m}, b=10 \mathrm{~cm}, \nu_{b}=$ 6.2, and $\gamma=2$ (see Ref. [5]), the smooth-approximation threshold is found to be an order of magnitude higher than the design intensity, $N_{\text {th }}=2 \times 10^{15}$, while the design intensity $N=2 \times 10^{14}$.

For the present design values of the Very Large Hadron Collider [6] with the circumference $233 \mathrm{~km}$, the betatron tune 214, the fractional betatron tune 0.5 (taken as an example), the vacuum chamber radius $0.9 \mathrm{~cm}$, and the relativistic factor $g=1000$ at injection, the threshold occurs at $4.9 \times 10^{14}$ protons, i.e., two times less than the design number of protons.

In reality, the threshold could be smaller than the smooth approximation result of Eq. (17), depending on beta function beating and the wake localization; see Eq. (12).

\section{CONCLUSION}

The drift instability of the closed orbit described here can be dangerous for high-intensity rings. The instability rate is highest when the working point is chosen slightly above integer resonance, where the conventional betatron modes for the resistive wall impedance are most stable. There are no chromaticity effects or Landau damping for the drift modes, because they are not coupled to the betatron oscillations. High-intensity machines can be examined theoretically for the closed orbit instability by solving the dispersion equation derived here for arbitrary lattice functions and impedance distribution.

\section{ACKNOWLEDGMENTS}

The authors thank J. Wei for his interest and support of this work, and A. Shishlo for useful discussions. 
[1] A. W. Chao, Physics of Collective Beam Instabilities in High Energy Accelerators (Wiley, New York, 1993), p. 172.

[2] D. A. Edwards and M. J. Syphers, An Introduction to the Physics of High Energy Accelerators (Wiley, New York, 1993), p. 92.

[3] V. Lebedev, "Control of Transverse Multibunch Instabili- ties in the First Stage of the VLHC," SLAC, 2001 (unpublished).

[4] S. Heifets et al., SLAC Report No. SLAC/AP-99, 1995.

[5] V. Danilov et al., Oak Ridge National Laboratory SNS Technical Note No. 14, 2001.

[6] Fermilab Report No. Fermilab-TM-2149, 2001. 